OPEN ACCESS

Edited by:

Linda Reiling,

Burnet Institute, Australia

Reviewed by:

Friedrich Frischknecht,

Heidelberg University, Germany

*Correspondence:

Takafumi Tsuboi

tsuboi.takafumi.mb@ehime-u.ac.jp

${ }^{\dagger}$ Present address:

Bernard N. Kanoi,

Centre for Research in Infectious Diseases, Directorate of Research and Innovation, Mount Kenya University,

Thika, Kenya

Specialty section:

This article was submitted to

Parasite and Host,

a section of the journal

Frontiers in Cellular and Infection

Microbiology

Received: 30 October 2021 Accepted: 16 November 2021 Published: 30 November 2021

Citation:

Takashima E, Tachibana M, Morita M, Nagaoka $H$,

Kanoi BN and Tsuboi T

(2021) Identification of Novel

Malaria Transmission-Blocking Vaccine Candidates.

Front. Cell. Infect. Microbiol. 11:805482.

doi: 10.3389/fcimb.2021.805482

\section{Identification of Novel Malaria Transmission-Blocking Vaccine Candidates}

\author{
Eizo Takashima ${ }^{1}$, Mayumi Tachibana ${ }^{2}$, Masayuki Morita ${ }^{1}$, Hikaru Nagaoka ${ }^{1}$, \\ Bernard N. Kanoi ${ }^{1+}$ and Takafumi Tsuboi ${ }^{3 *}$
}

${ }^{1}$ Division of Malaria Research, Proteo-Science Center, Ehime University, Matsuyama, Japan, ${ }^{2}$ Division of Molecular Parasitology, Proteo-Science Center, Ehime University, Toon, Japan, ${ }^{3}$ Division of Cell-Free Sciences, Proteo-Science Center, Ehime University, Matsuyama, Japan

Control measures have significantly reduced malaria morbidity and mortality in the last two decades; however, the downward trends have stalled and have become complicated by the emergence of COVID-19. Significant efforts have been made to develop malaria vaccines, but currently only the RTS,S/AS01 vaccine against Plasmodium falciparum has been recommended by the WHO, for widespread use among children in sub-Saharan Africa. The efficacy of RTS,S/AS01 is modest, and therefore the development of more efficacious vaccines is still needed. In addition, the development of transmission-blocking vaccines (TBVs) to reduce the parasite transmission from humans to mosquitoes is required toward the goal of malaria elimination. Few TBVs have reached clinical development, and challenges include low immunogenicity or high reactogenicity in humans. Therefore, novel approaches to accelerate TBV research and development are urgently needed, especially novel TBV candidate discovery. In this mini review we summarize the progress in TBV research and development, novel TBV candidate discovery, and discuss how to accelerate novel TBV candidate discovery.

Keywords: immuno-profiling, malaria, Plasmodium, reverse vaccinology, transmission-blocking vaccine (TBV), wheat germ cell-free system (WGCFS)

\section{INTRODUCTION}

Malaria continues to be responsible for a substantial global health burden, with 409,000 malarial deaths reported in 2019 (WHO, 2020). From 2000 to 2015, malaria morbidity and mortality were significantly reduced; however, the decreasing trend stalled between 2015 and 2019 and was further complicated by the emergence of COVID-19 (Wang et al., 2020; WHO, 2020). Therefore, the

\footnotetext{
Abbreviations: AnAPN1, anopheline alanyl aminopeptidase N 1; BDES, baculovirus dual expression system; E., Escherichia; EPA, ExoProtein A from Pseudomonas aeruginosa; HAP2/GCS1, Hapless 2/Generative Cell Specific 1; IFA, indirect immunofluorescence assay; MiGS, microgamete surface protein; P., Plasmodium; PH, pleckstrin homology; PSOP, putative secreted ookinete protein; SMFA, standard membrane feeding assay; TBA, transmission-blocking activity; TBV, transmissionblocking vaccine; TRA, transmission-reducing activity; WGCFS, wheat germ cell-free system; WHO, The World Health Organization.
} 
control and eventual eradication of this disease relies on the development of a highly effective malaria vaccine.

Malaria vaccines can be categorized into three groups, each targeting distinct parasite developmental stages: pre-erythrocytic (sporozoite and liver), asexual erythrocytic, and sexual transmission stages. The renewed Malaria Vaccine Technology Roadmap proposes two main objectives by 2030 for the development of new malaria vaccines targeting both Plasmodium falciparum and Plasmodium vivax: i) vaccines with protective efficacy of at least $75 \%$ against clinical malaria, and ii) vaccines that reduce transmission of the parasite (Group, 2013; Moorthy et al., 2013). A leading malaria vaccine RTS,S/AS01 was the first malaria vaccine to enter Phase III clinical trials and shows modest efficacy against clinical falciparum malaria (RTS, 2015) with short durability (White et al., 2015). It is currently being evaluated in a large pilot implementation program in Ghana, Kenya, and Malawi since 2019 (Adepoju, 2019). The vaccine reduced severe malaria by about $30 \%$ in the first 2 years of the program (Vogel, 2021). Based on this, the World Health Organization (WHO) is now recommending widespread use of the RTS,S/AS01 malaria vaccine among children in sub-Saharan Africa and in other regions with moderate to high P. falciparum malaria transmission (Vogel, 2021).

Since the RTS,S/AS01 vaccine efficacy is modest, the development of more efficacious vaccines is still needed. A number of second-generation malaria vaccines are in clinical trials, such as R21/Matrix-M (Datoo et al., 2021). However, the above mentioned two malaria vaccines are classified as preerythrocytic stage vaccines. Therefore, the development of erythrocytic stage vaccines to reduce morbidity and mortality, and transmission-blocking vaccines (TBVs) to reduce parasite transmission from humans to mosquitoes, are required to reach the Roadmap goals.

\section{MALARIA TRANSMISSION-BLOCKING VACCINES (TBVS)}

The principle of malaria TBVs is that antibodies against antigen(s) expressed on the sexual stages of the malaria parasite gametocyte/gamete/zygote/ookinete - reduce the numbers of oocysts in mosquito vectors when fed with gametocytes (Huff et al., 1958; Carter and Chen, 1976; Gwadz, 1976). The advantages of TBVs are summarized as follows (Tsuboi et al., 2003; Miura et al., 2019; Duffy, 2021): i) TBV candidates tend to be less polymorphic than blood- or pre-erythrocytic-stage antigens, presumably due to lower immune pressure driving evolutionary diversity; ii) the absolute number of parasites targeted by TBVs is small, usually $<10-100$ oocysts per mosquito in nature, and represent a biological bottleneck in the malaria parasite lifecycle; and iii) TBVs might help to prevent the spread of emerging drugresistant parasites (Dondorp et al., 2009; Balikagala et al., 2021) and future vaccine-escape mutants.

Target antigens include proteins expressed on the surface of gametocytes/gametes/zygotes/ookinetes; such as the characterized proteins P230, P48/45, P28, and P25 (Carter and Kaushal, 1984;
Kumar and Carter, 1985; Vermeulen et al., 1985). To initiate vaccine research, the antigens in human malaria parasites were identified in the pre-genomic era; namely, Pfs25 (Kaslow et al., 1988; Kaslow et al., 1994), Pfs28 (Duffy and Kaslow, 1997), Pfs48/ 45 (Kocken et al., 1993; Outchkourov et al., 2008), and Pfs230 (Williamson et al., 1993; Williamson et al., 1995) from $P$. falciparum; and their orthologs in P. vivax, Pvs25 and Pvs28 (Tsuboi et al., 1998; Hisaeda et al., 2000). Soon after whole genome information became accessible, Pvs48/45 (Arevalo-Herrera et al., 2015; Tachibana et al., 2015) and Pvs230 (Tachibana et al., 2012) were also characterized as TBV candidates (Table 1).

Researchers have faced a number of difficulties to express TBV antigens with native conformations (Miura et al., 2019), using a variety of protein expression systems (Patel and Tolia, 2021). Antibodies raised against individual antigens needed to be tested in an ex vivo efficacy assay; specifically, the standard membrane feeding assay (SMFA) wherein laboratory-reared Anopheles mosquitoes are fed on in vitro cultured $P$. falciparum gametocytes along with test antisera or purified antibodies, and counts of midgut wall oocysts as a measure of the degree of transmission-blocking activity (Miura et al., 2013a).

\section{TBV DEVELOPMENT EFFORTS TO DATE}

After decades of efforts, the most advanced P. falciparum TBV antigens in the clinical pipeline remain the first identified antigens: Pfs25 expressed on the surface of zygotes/ookinetes in the mosquito and classified as a post-fertilization antigen, and Pfs48/ 45 and Pfs230 expressed on the surface of blood-circulating gametocytes and gametes in the mosquito and classified as prefertilization antigens. In addition, a mosquito midgut protein, anopheline alanyl aminopeptidase N 1 (AnAPN1) (Armistead et al., 2014), is under development as a TBV candidate in preclinical developmental studies (Bender et al., 2021) (Table 1, Figure 1). As transmission-blocking immunity is mostly antibody-mediated (de Jong et al., 2020), TBV development efforts focus on inducing potent antibodies that are sustained at effective transmission-blocking levels for at least one transmission season. Based on these requirements, extensive efforts towards the clinical development of $P$. falciparum TBVs continue to date. Recently, phase 1 trials of $P$. falciparum TBV based upon Pfs25/ Alhydrogel (Alum) have been reported. These studies used Pfs25EPA: Pfs25 conjugated with a recombinant detoxified ExoProtein A from Pseudomonas aeruginosa (EPA), formulated with Alum, and tested in adults in the USA (Talaat et al., 2016) and Mali (Sagara et al., 2018). The vaccine was generally well-tolerated; however, the functional activity of the anti-Pfs25 antibodies induced were modest, and antibody titers decreased rapidly.

To improve functional immunogenicity and durability, the same group performed a phase 1 trial of the pre-fertilization TBV antigen Pfs 230 alone or in combination with Pfs 25 in USA adults. Pfs25-EPA/Alum and Pfs230D1M [amino acid 542-736 (MacDonald et al., 2016)]-EPA/Alum induced similar serum functional activity in mice, but Pfs230D1M-EPA induced significantly greater activity in rhesus monkeys. In USA adults, 
TABLE 1 | Discovery of malaria transmission-blocking vaccine antigens with publication years ${ }^{a}$.

\begin{tabular}{|c|c|c|c|c|c|c|}
\hline Antigen $^{b}$ & Yearc $^{c}$ & $\begin{array}{c}\text { Target } \\
\text { parasite }^{d}\end{array}$ & Developmental stage $^{\mathrm{e}}$ & Discovery $^{\mathbf{f}}$ & Expression system ${ }^{g}$ & Reference \\
\hline \multicolumn{7}{|l|}{ Pre-Genomic Era } \\
\hline Pfs25 & 1988 & $P f$ & Zygote/ookinete & Gene & - & (Kaslow et al., 1988) \\
\hline Pfs25 & 1994 & $P f$ & Zygote/ookinete & TRA & Yeast & (Kaslow et al., 1994) \\
\hline Pfs28 & 1997 & $P f$ & Zygote/ookinete & Gene/TRA & Yeast & (Duffy and Kaslow, 1997) \\
\hline Pfs48/45 & 1993 & Pf & Gametocyte/gamete & Gene & - & (Kocken et al., 1993) \\
\hline Pfs48/45 & 2008 & $P f$ & Gametocyte/gamete & TRA & Bacteria & (Outchkourov et al., 2008) \\
\hline Pfs230 & 1993 & $P f$ & Gametocyte/gamete & Gene & - & (Williamson et al., 1993) \\
\hline Pfs230 & 1995 & $P f$ & Gametocyte/gamete & TRA & Bacteria & (Williamson et al., 1995) \\
\hline Pvs25 \& Pvs28 & 1998 & PV & Zygote/ookinete & Gene & - & (Tsuboi et al., 1998) \\
\hline Pvs25 \& Pvs28 & 2000 & PV & Zygote/ookinete & TRA & Yeast & (Hisaeda et al., 2000) \\
\hline \multicolumn{7}{|l|}{ Post-Genomic Era } \\
\hline HAP2/GCS1 & 2008 & $P b$ & Gamete & Gene & - & (Hirai et al., 2008; Liu et al., 2008) \\
\hline HAP2/GCS1 & 2009 & $P b$ & Gamete & TRA & Bacteria & (Blagborough and Sinden, 2009) \\
\hline HAP2/GCS1 & 2013 & Pf & Gamete & TRA & WGCFS & (Miura et al., 2013b) \\
\hline HAP2/GCS1 & 2017 & $P b, P f$ & Gamete & TRA & Peptide & (Angrisano et al., 2017) \\
\hline HAP2/GCS1 & 2020 & PV & Gamete & TRA & Baculovirus & (Qiu et al., 2020) \\
\hline Pvs230 & 2012 & PV & Gametocyte/gamete & TRA & DNA & (Tachibana et al., 2012) \\
\hline Pvs48/45, Pvs47 & 2015 & PV & Gametocyte/gamete & TRA & DNA, Bacteria & $\begin{array}{l}\text { (Arevalo-Herrera et al., 2015; } \\
\text { Tachibana et al., 2015) }\end{array}$ \\
\hline Pfs47 & 2010 & $P f$ & Gametocyte/gamete & Gene & - & (van Schaijk et al., 2006) \\
\hline Pfs47 & 2018 & $P f$ & Gametocyte/gamete & TRA & Bacteria & (Canepa et al., 2018) \\
\hline AnAPN1 & 2014 & $P f, P V$ & Anopheles midgut & Gene/TRA & Drosophila S2 & (Armistead et al., 2014) \\
\hline PbPSOP12 & 2015 & $\mathrm{~Pb}$ & Gamete - ookinete & TRA & BDES & (Sala et al., 2015) \\
\hline $\mathrm{PbPH}$ & 2016 & $P b$ & Gamete - ookinete & TRA & Bacteria & (Kou et al., 2016) \\
\hline PbPSOP7, 25 \& 26 & 2016 & $P b$ & Ookinete & TRA & Bacteria & (Zheng et al., 2016) \\
\hline PbPSOP25 & 2017 & & & & & (Zheng et al., 2017) \\
\hline Pb51 & 2017 & $P b$ & Gametocyte - ookinete & TRA & Bacteria & (Wang et al., 2017) \\
\hline Pbg37 & 2018 & $P b$ & Gametocyte - zygote & TRA & Bacteria & (Liu et al., 2018) \\
\hline PyMiGS & $\begin{array}{l}2018 \\
2020\end{array}$ & Py & Gametocyte/gamete & TRA & WGCFS & $\begin{array}{l}\text { (Tachibana et al., 2018a; Tachibana } \\
\text { et al. 2018b: Tachibana et al. 2020) }\end{array}$ \\
\hline $\mathrm{Pb} 22$ & 2021 & $P b$ & Gamete - ookinete & TRA & Bacteria & (Liu et al., 2021) \\
\hline
\end{tabular}

asummary of the TBV antigen discovery efforts in which significant TRA has been confirmed.

${ }^{b}$ Antigen, abbreviated names of TBV antigens.

${ }^{c}$ Year, year of publication.

${ }^{d}$ Target parasite, Pf, Plasmodium falciparum; PV, P. vivax; Pb, P. berghei; Py, P. yoelii.

${ }^{e}$ Developmental stage, parasite developmental stage(s) in which target the antigen is expressed.

${ }^{f}$ Discovery, Gene, target gene discovered; TRA, antigens specific antibodies with confirmed transmission reducing/blocking activity identified..

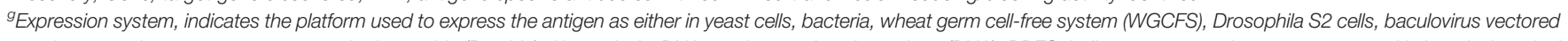

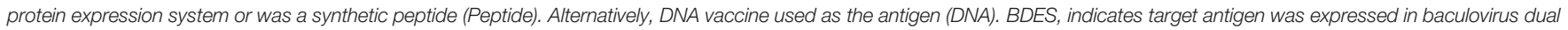
expression system.

two vaccine doses induced functional activity in Pfs230D1MEPA/Alum volunteers, but no significant activity in Pfs25-EPA vaccine recipients, and combination with Pfs25-EPA did not increase functional activity over Pfs230D1M-EPA alone. The research group concluded that the functional activity of Pfs230D1M-EPA is significantly superior to that of Pfs25-EPA (Healy et al., 2021). For more information about the clinical development of these falciparum TBVs, please refer to two recent review articles (Miura et al., 2019; Duffy, 2021). In addition to the above TBV development efforts, novel TBV candidate discovery is required to accelerate the success in TBV development.

\section{POST-GENOME NOVEL TBV CANDIDATE DISCOVERY}

The goal of identifying new vaccine candidates for both $P$. falciparum and $P$. vivax is aided by whole genome information accessible since 2003 at the malaria genome database (PlasmoDB). The database has been useful to identify vaccine candidates from asexual-blood (Kanoi et al., 2021) and pre-erythrocytic (Bettencourt, 2020) stages. However, the rational selection and prioritization of TBV candidates from the database has yet to be fully explored (Miura et al., 2019). Extensive proteome and transcriptome data from sexual-stage malaria parasites is now available (Lasonder et al., 2016; Meerstein-Kessel et al., 2018) to inform in silico TBV candidate discovery. In the following sections we summarize the recent achievements for the discovery of the activities and candidate antigens discovered in the post-genomic era (Table 1, Figure 1).

\section{RODENT MALARIA MODELS FOR NOVEL TBV CANDIDATE DISCOVERY}

Most of the TBV candidates investigated to date have orthologs in rodent malaria parasites, and thus the rodent malaria models 


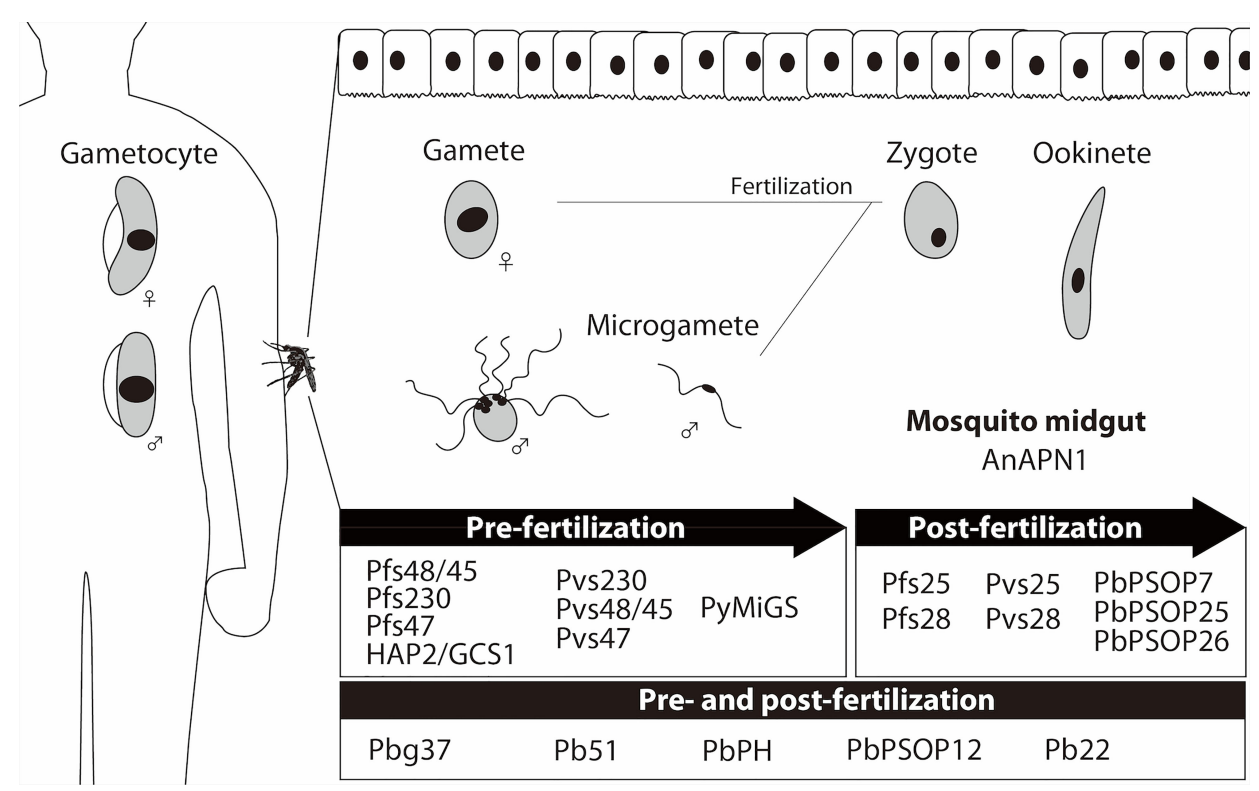

FIGURE 1 | Expression of malaria transmission-blocking vaccine (TBV) target antigens. Sexual developmental stages of malaria parasites in humans (gametocytes) and mosquitoes (gametes, zygotes, and ookinetes) are schematically presented. The TBV candidate antigens (Table 1) are categorized as pre-fertilization antigens (mainly expressed in the sexual stages of parasites before fertilization), and post-fertilization antigens (mainly expressed in the sexual stages of parasites after fertilization). Mosquito midgut antigen, AnAPN1, is also presented as a TBV candidate.

are useful for the discovery and characterization of novel TBV candidates. In the last decade several potential TBV candidates have been identified using rodent malaria models. The general strategy of these studies is to select candidate genes from the PlasmoDB according to the following criteria: i) genes must be specifically expressed in sexual-stages; ii) they must share orthologs with human parasites, in particular P. falciparum and $P$. vivax; and iii) the presence of a predicted signal peptide with/without transmembrane domain(s) or a GPI-anchor, indicating possible protein export and exposure to inhibitory antibodies. Candidate TBV genes are then expressed in one or more recombinant protein expression systems, followed by immunization of mice. To test efficacy, immunized mice are infected with rodent malaria parasites and then mosquitoes are fed directly on these mice; termed a direct feeding assay. The transmission-blocking activity (TBA) is expressed as a percent reduction of the prevalence of infected mosquitoes; and transmission-reducing activity (TRA) is expressed as a percent reduction of oocyst density.

The majority of such studies were conducted with $P$. berghei rodent parasites because of the ease for genetic manipulation, such as the knockout of candidate genes for functional characterization of novel TBV candidates. Most such activities are listed in Table 1, classified in the post-genomic era, and following are descriptions of examples of post-genomic studies.

As the first examples, a group actively working on novel TBV candidate discovery using the $P$. berghei model identified a conserved $P$. berghei protein, $\mathrm{PbPH}$, containing a pleckstrin homology $(\mathrm{PH})$ domain. By indirect immunofluorescence assay (IFA) $\mathrm{PbPH}$ localized on the surface of gametes/zygotes/ ookinetes. Mice were immunized with recombinant $\mathrm{PbPH}$ expressed in E. coli and mosquitoes fed on the immunized mice showed a $48 \%$ TRA (Kou et al., 2016). Similarly, the same group selected $P$. berghei ookinete-stage proteins, Putative Secreted Ookinete Protein (PbPSOP25), PbPSOP26, and PbPSOP7, for evaluation of their transmission-blocking potentials. Antisera against these bacterially expressed partial recombinant proteins recognized the ookinete surface. Mosquitoes fed on immunized mice showed significant TRAs (60\% to $71 \%$ ) (Zheng et al., 2016). Mice immunized with fulllength recombinant PSOP25 expressed in E. coli and those receiving passive transfer of an anti-rPSOP25 mAb showed significant TRAs by $66 \%$ and $63 \%$, respectively (Zheng et al., 2017).

The conserved Plasmodium gene, $\mathrm{Pb} 51$, was identified in $P$. berghei through PlasmoDB using gene expression and protein localization criteria. A partial domain of $\mathrm{Pb} 51$ was expressed in E. coli and mice were immunized. By IFA Pb51 was expressed in schizonts/gametocytes/ookinetes of P. berghei. Mice immunized with the recombinant $\mathrm{Pb} 51$ showed 55\% TRA in direct feeding assays (Wang et al., 2017). Using a similar approach, the same group characterized a protein of $37 \mathrm{kDa}$ preferentially expressed in gametocytes in P. berghei (Pbg37). A recombinant Pbg37 (rPbg37) was expressed in bacteria and antibody was generated in mice. IFA showed surface expression of Pbg37 on gametes/ zygotes. The rPbg37-immunized mice had a significant TRA (49\%) (Liu et al., 2018). Similarly, a gamete/ookinete surface protein of $P$. berghei, $\mathrm{Pb} 22$, was identified and recombinant $\mathrm{Pb} 22$ was expressed in E. coli. The $\mathrm{Pb} 22$-immunised mice had a significant TRA (93.5-99.6\%) (Liu et al., 2021). 
The $P$. berghei ookinete-stage protein, PbPSOP12, was identified based upon annotation as a putative secreted protein and then expressed using the baculovirus dual expression system (BDES). Mouse antibodies against BDES-PbPSOP12 recognized the surface of gametes/ookinetes. Immunization of mice with BDES-PbPSOP12 conferred modest TRA (53\%) (Sala et al., 2015).

Our lab has accumulated a number of experiences using the Plasmodium yoelii rodent malaria parasite as a suitable model for TBV study; such as the identification of Pfs25 and Pfs28 orthologs in P. yoelii, Pys25 and Pys28 (Tsuboi et al., 1997a; Tsuboi et al., 1997b; Tsuboi et al., 1997c). Recently we identified a novel TBV candidate, $P$. yoelii microgamete surface protein (PyMiGS), using a similar approach as mentioned above for the $P$. berghei studies. PyMiGS is a protein expressed in the osmiophilic body of male gametocytes of $P$. yoelii and is translocated to the surface of microgametes. Potent TRA (>99\%) was observed in mosquitoes fed on mice passively immunized with antibodies against recombinant full-length PyMiGS expressed using a wheat germ cell-free protein expression system (WGCFS) (Tachibana et al., 2018a). Mice actively immunized with the recombinant full-length PyMiGS conferred $>99 \%$ TRA using direct mosquito feeding (Tachibana et al., 2018b), and the major epitopes for transmission-blocking antibodies were within the C-terminal region of PyMiGS (Tachibana et al., 2020).

Although the $P$. berghei and $P$. yoelii rodent malaria models are useful to identify novel TBV candidates, results between the models may differ. For example, when we characterized the phenotype of a PyMiGS gene deletion mutant ( $\triangle$ PyMiGS), the ookinete formation efficiency of $\triangle \mathrm{PyMiGS}$ was significantly impaired (Tachibana et al., 2018a). Contrary, ookinete formation of the gene deletion mutant of the P. berghei ortholog of PyMiGS (PBANKA_1449000) was not impaired (Kehrer et al., 2016). Accordingly, although the usefulness of the rodent models is clear, careful consideration is also required.

Candidates identified in the rodent malaria studies should be evaluated with $P$. falciparum orthologs. For example, a conserved male gamete sterility gene, HAP2/GCS1 (Hapless 2/Generative Cell Specific 1), was initially identified as an essential protein for the fusion of male and female gametes of $P$. berghei (Hirai et al., 2008; Liu et al., 2008). Genetic disruption of the hap2 locus revealed that parasite fertilization is inhibited, and anti-PbHAP2 sera showed TRA by up to $81 \%$ (Blagborough and Sinden, 2009). Mosquitoes fed on mice immunized with PbHAP2 cd loop peptide showed 59\% TRA in P. berghei and the corresponding TRA in P. falciparum was 76\% (Angrisano et al., 2017). We also demonstrated strong transmission-blocking activity of mouse antibody against recombinant $P$. falciparum HAP2 protein and concluded the antigen to be a novel TBV candidate (Miura et al., 2013b). Recently, recombinant $P$. vivax HAP2 was expressed in a baculovirus expression system, and rabbit antibody induced significant TRA (40\% to $90 \%$ ) against $P$. vivax field isolates in Anopheles dirus (Qiu et al., 2020).

The gametocyte/gamete protein $\mathrm{P} 47$ is another example of experimental system-specific differences. When the $p 47$ gene was disrupted, a strong reduction of female fertility was observed in
P. berghei (van Dijk et al., 2010), but not in P. falciparum (van Schaijk et al., 2006), and anti-Pfs47 mAbs showed no efficacy in P. falciparum SMFA (van Schaijk et al., 2006). Similarly, mAbs and polyclonal antibodies against a full-length recombinant Pfs47 protein did not show efficacy in SMFA. However, antibodies against a part of domain 2 in Pfs47 did demonstrate significant TRA (Canepa et al., 2018). Further characterization revealed that when mice were immunized with the full-length protein, almost no antibody was induced against the critical domain 2. Therefore, it is possible that other potential TBV candidates were overlooked in previous studies (Miura et al., 2019); and improvement of antigen design and vaccine formulations with existing TBV candidates, and expansion of the repertoire of novel TBV candidates, are necessary to accelerate TBV development.

\section{NOVEL TBV CANDIDATE DISCOVERY DIRECTLY USING HUMAN MALARIA PARASITES}

In P. falciparum only two studies on genome-wide novel TBV candidate discovery have been reported to date. One is a reverse vaccinology approach by Nikolaeva et al. (Nikolaeva et al., 2020). They identified a panel of potential TBV candidate genes from PlasmoDB by selecting with a sexual-stage specific expression profile. After a logical in-silico process to narrow down the candidate list, they expressed 21 recombinant proteins using a human embryonic kidney cell (HEK293) expression system. Twelve proteins were successfully expressed, and mouse antibodies against the recombinant proteins were tested by SMFA. However, none of the novel TBV candidates showed TRA. It is possible that the heterologous human cell expression system resulted in aberrant glycosylation patterns compared with Plasmodium, which has a minimal glycosylation machinery, and the resulting antibodies did not recognize native Plasmodium protein (Kanoi et al., 2021).

The other is a larger-scale trial of immuno-profiling of naturally occurring antibody-mediated TRA (Stone et al., 2018). Bioinformatically selected 315 proteins were expressed using an $E$. coli cell-free system, and correctly-folded wellcharacterized recombinant $\mathrm{Pfs} 48 / 45$ and $\mathrm{Pfs} 230$ proteins were used as positive controls. They assessed antibody responses in 648 African plasma samples with TRA measured by SMFA, and those with high $(\geq 90 \%, n=22)$ or low $(<10 \%, n=254)$ TRA were used for the immuno-profiling. Forty-three out of 315 proteins in addition to Pfs230 and Pfs48/45 had significantly higher antibody levels in plasmas with high TRA. After additional consideration on the protein expression levels in gametocytes, and the presence of a signal peptide or a transmembrane domain, 13 out of the 43 proteins were selected as possible TBV candidates. Although the strategy of this work is convincing, to date they have not validated whether any of the 13 novel TBV candidates could induce transmission-blocking antibodies in immunized animals. In addition, since the reacted human 
antibodies were likely to recognize only linear epitopes of the tested antigens, because the proteins were expressed in E. coli, this work may have missed promising candidates which have conformational TRA epitopes/antigens (Miura et al., 2019). Finally, the approach might not identify TBV candidates whose protein expression is solely in the mosquito and not in gametocytes.

Additional gametocyte-specific gene discovery efforts have been published (Ikadai et al., 2013; Chawla et al., 2021; Muthui et al., 2021); although antigen expression, immunization, and TRA assessment of the antibodies are not completed.

\section{KEY MESSAGES TO THE NOVEL TBV CANDIDATE DISCOVERY}

The clinical development of $P$. falciparum TBV have advanced to Phase 2 clinical trials (Duffy, 2021). However, those efforts have focused only on the leading candidates - Pfs25, Pfs230, and Pfs48/45 - which were identified in the pre-genome era (Miura et al., 2019). To accelerate TBV research and development in the post-genome era, genome-wide discovery of novel TBV candidates by both immuno-profiling and reverse vaccinology approaches are essential. A key message learned from the pioneering post-genome TBV candidate discovery approaches is that it is crucial to select an expression system with the capability of producing large numbers of correctly-folded malaria recombinant proteins, and without artificial glycosylation. We have been using the WGCFS to express a number of high-quality recombinant proteins of both $P$. falciparum and $P$. vivax; and to produce comprehensive genome-wide protein libraries useful for novel malaria vaccine and sero-marker candidate discovery projects (Morita et al., 2017; Kanoi et al., 2018; Longley et al., 2020;

\section{REFERENCES}

Adepoju, P. (2019). RTS,S Malaria Vaccine Pilots in Three African Countries. Lancet 393, 1685. doi: 10.1016/S0140-6736(19)30937-7

Angrisano, F., Sala, K. A., Da, D. F., Liu, Y., Pei, J., Grishin, N. V., et al. (2017). Targeting the Conserved Fusion Loop of HAP2 Inhibits the Transmission of Plasmodium Berghei and Falciparum. Cell Rep. 21, 2868-2878. doi: 10.1016/ j.celrep.2017.11.024

Arevalo-Herrera, M., Vallejo, A. F., Rubiano, K., Solarte, Y., Marin, C., Castellanos, A., et al. (2015). Recombinant Pvs48/45 Antigen Expressed in E. Coli Generates Antibodies That Block Malaria Transmission in Anopheles Albimanus Mosquitoes. PloS One 10, e0119335. doi: 10.1371/journal.pone.0119335

Armistead, J. S., Morlais, I., Mathias, D. K., Jardim, J. G., Joy, J., Fridman, A., et al. (2014). Antibodies to a Single, Conserved Epitope in Anopheles APN1 Inhibit Universal Transmission of Plasmodium Falciparum and Plasmodium Vivax Malaria. Infect. Immun. 82, 818-829. doi: 10.1128/IAI.01222-13

Balikagala, B., Fukuda, N., Ikeda, M., Katuro, O. T., Tachibana, S. I., Yamauchi, M., et al. (2021). Evidence of Artemisinin-Resistant Malaria in Africa. N. Engl. J. Med. 385, 1163-1171. doi: 10.1056/NEJMoa2101746

Bender, N. G., Khare, P., Martinez, J., Tweedell, R. E., Nyasembe, V. O., LopezGutierrez, B., et al. (2021). Immunofocusing Humoral Immunity Potentiates the Functional Efficacy of the AnAPN1 Malaria Transmission-Blocking Vaccine Antigen. NPJ Vaccines 6, 49. doi: 10.1038/s41541-021-00309-4
Kanoi et al., 2021). Therefore, following genome-wide gametocyte stage protein expression by WGCFS, these proteins can then be used in immuno-profiling approaches using human plasma with known TRA, to identify novel transmissionblocking antigens (Ntege et al., 2017; Miura et al., 2019; Kanoi et al., 2021). To this end it is also essential to obtain wellcharacterized plasma samples from infected individuals who carry transmission-reducing antibodies.

\section{AUTHOR CONTRIBUTIONS}

All authors listed have made a substantial, direct, and intellectual contribution to the work and approved it for publication.

\section{FUNDING}

Some of the work presented here was partially supported by JSPS KAKENHI Grant (JP18H02651, JP20H03481, JP21H02724, JP21K06990, JP21KK0138), Global Health Innovative Technology (GHIT) Fund (grant \# G2019-111, G2019-205), and the Takeda Science Foundation. BNK is an EDCTP Fellow under EDCTP2 program supported by the European Union (grant TMA2020CDF-3203-EndPAMAL). The funding sources had no role in study design, collection, analysis, interpretation of data, and publication.

\section{ACKNOWLEDGMENTS}

We thank Dr. Thomas J. Templeton for critical reading of the manuscript.

Bettencourt, P. (2020). Current Challenges in the Identification of PreErythrocytic Malaria Vaccine Candidate Antigens. Front. Immunol. 11:190. doi: 10.3389/fimmu.2020.00190

Blagborough, A. M., and Sinden, R. E. (2009). Plasmodium Berghei HAP2 Induces Strong Malaria Transmission-Blocking Immunity In Vivo and In Vitro. Vaccine 27, 5187-5194. doi: 10.1016/j.vaccine.2009.06.069

Canepa, G. E., Molina-Cruz, A., Yenkoidiok-Douti, L., Calvo, E., Williams, A. E., Burkhardt, M., et al. (2018). Antibody Targeting of a Specific Region of Pfs47 Blocks Plasmodium Falciparum Malaria Transmission. NPJ Vaccines 3, 26. doi: 10.1038/s41541-018-0065-5

Carter, R., and Chen, D. H. (1976). Malaria Transmission Blocked by Immunisation With Gametes of the Malaria Parasite. Nature 263, 57-60. doi: $10.1038 / 263057 \mathrm{a} 0$

Carter, R., and Kaushal, D. C. (1984). Characterization of Antigens on Mosquito Midgut Stages of Plasmodium Gallinaceum. III. Changes in Zygote Surface Proteins During Transformation to Mature Ookinete. Mol. Biochem. Parasitol 13, 235-241. doi: 10.1016/0166-6851(84)90116-6

Chawla, J., Oberstaller, J., and Adams, J. H. (2021). Targeting Gametocytes of the Malaria Parasite Plasmodium Falciparum in a Functional Genomics Era: Next Steps. Pathogens 10:346. doi: 10.3390/pathogens10030346

Datoo, M. S., Natama, M. H., Some, A., Traore, O., Rouamba, T., Bellamy, D., et al. (2021). Efficacy of a Low-Dose Candidate Malaria Vaccine, R21 in Adjuvant Matrix-M, With Seasonal Administration to Children in Burkina Faso: A 
Randomised Controlled Trial. Lancet 397, 1809-1818. doi: 10.1016/S01406736(21)00943-0

De Jong, R. M., Tebeje, S. K., Meerstein-Kessel, L., Tadesse, F. G., Jore, M. M., Stone, W., et al. (2020). Immunity Against Sexual Stage Plasmodium Falciparum and Plasmodium Vivax Parasites. Immunol. Rev. 293, 190-215. doi: $10.1111 /$ imr. 12828

Dondorp, A. M., Nosten, F., Yi, P., Das, D., Phyo, A. P., Tarning, J., et al. (2009). Artemisinin Resistance in Plasmodium Falciparum Malaria. N. Engl. J. Med. 361, 455-467. doi: 10.1056/NEJMoa0808859

Duffy, P. E. (2021). Transmission-Blocking Vaccines: Harnessing Herd Immunity for Malaria Elimination. Expert Rev. Vaccines 20, 185-198. doi: 10.1080/ 14760584.2021 .1878028

Duffy, P. E., and Kaslow, D. C. (1997). A Novel Malaria Protein, Pfs28, and Pfs25 are Genetically Linked and Synergistic as Falciparum Malaria TransmissionBlocking Vaccines. Infect. Immun. 65, 1109-1113. doi: 10.1128/iai.65.3.11091113.1997

Group, M. V. F. (2013). Malaria Vaccine Technology Roadmap (Geneva, Switzerland: WHO Press).

Gwadz, R. W. (1976). Successful Immunization Against the Sexual Stages of Plasmodium Gallinaceum. Science 193, 1150-1151. doi: 10.1126/ science. 959832

Healy, S. A., Anderson, C., Swihart, B. J., Mwakingwe, A., Gabriel, E. E., Decederfelt, H., et al. (2021). Pfs230 Yields Higher Malaria TransmissionBlocking Vaccine Activity Than Pfs 25 in Humans But Not Mice. J. Clin. Invest. 131, e146221. doi: 10.1172/JCI146221

Hirai, M., Arai, M., Mori, T., Miyagishima, S. Y., Kawai, S., Kita, K., et al. (2008). Male Fertility of Malaria Parasites Is Determined by GCS1, a Plant-Type Reproduction Factor. Curr. Biol. 18, 607-613. doi: 10.1016/j.cub.2008.03.045

Hisaeda, H., Stowers, A. W., Tsuboi, T., Collins, W. E., Sattabongkot, J. S., Suwanabun, N., et al. (2000). Antibodies to Malaria Vaccine Candidates Pvs25 and Pvs28 Completely Block the Ability of Plasmodium Vivax to Infect Mosquitoes. Infect. Immun. 68, 6618-6623. doi: 10.1128/ iai.68.12.6618-6623.2000

Huff, C. G., Marchbank, D. F., and Shiroishi, T. (1958). Changes in Infectiousness of Malarial Gametocytes. II. Analysis of the Possible Causative Factors. Exp. Parasitol 7, 399-417. doi: 10.1016/0014-4894(58)90036-5

Ikadai, H., Shaw Saliba, K., Kanzok, S. M., Mclean, K. J., Tanaka, T. Q., Cao, J., et al. (2013). Transposon Mutagenesis Identifies Genes Essential for Plasmodium Falciparum Gametocytogenesis. Proc. Natl. Acad. Sci. U.S.A. 110, E1676-E1684. doi: 10.1073/pnas.1217712110

Kanoi, B. N., Nagaoka, H., Morita, M., Tsuboi, T., and Takashima, E. (2021). Leveraging the Wheat Germ Cell-Free Protein Synthesis System to Accelerate Malaria Vaccine Development. Parasitol Int. 80:102224. doi: 10.1016/ j.parint.2020.102224

Kanoi, B. N., Nagaoka, H., Morita, M., White, M. T., Palacpac, N. M. Q., Ntege, E. H., et al. (2018). Comprehensive Analysis of Antibody Responses to Plasmodium Falciparum Erythrocyte Membrane Protein 1 Domains. Vaccine 36, 6826-6833. doi: 10.1016/j.vaccine.2018.08.058

Kaslow, D. C., Bathurst, I. C., Lensen, T., Ponnudurai, T., Barr, P. J., and Keister, D. B. (1994). Saccharomyces Cerevisiae Recombinant Pfs 25 Adsorbed to Alum Elicits Antibodies That Block Transmission of Plasmodium Falciparum. Infect. Immun. 62, 5576-5580. doi: 10.1128/iai.62.12.5576-5580.1994

Kaslow, D. C., Quakyi, I. A., Syin, C., Raum, M. G., Keister, D. B., Coligan, J. E., et al. (1988). A Vaccine Candidate From the Sexual Stage of Human Malaria That Contains EGF-Like Domains. Nature 333, 74-76. doi: 10.1038/333074a0

Kehrer, J., Frischknecht, F., and Mair, G. R. (2016). Proteomic Analysis of the Plasmodium Berghei Gametocyte Egressome and Vesicular bioID of Osmiophilic Body Proteins Identifies Merozoite TRAP-Like Protein (MTRAP) as an Essential Factor for Parasite Transmission. Mol. Cell Proteomics 15, 2852-2862. doi: 10.1074/mcp.M116.058263

Kocken, C. H., Jansen, J., Kaan, A. M., Beckers, P. J., Ponnudurai, T., Kaslow, D. C., et al. (1993). Cloning and Expression of the Gene Coding for the Transmission Blocking Target Antigen Pfs48/45 of Plasmodium Falciparum. Mol. Biochem. Parasitol 61, 59-68. doi: 10.1016/0166-6851(93)90158-T

Kou, X., Zheng, W., Du, F., Liu, F., Wang, M., Fan, Q., et al. (2016). Characterization of a Plasmodium Berghei Sexual Stage Antigen PbPH as a New Candidate for Malaria Transmission-Blocking Vaccine. Parasit Vectors 9 , 190. doi: 10.1186/s13071-016-1459-8
Kumar, N., and Carter, R. (1985). Biosynthesis of Two Stage-Specific Membrane Proteins During Transformation of Plasmodium Gallinaceum Zygotes Into Ookinetes. Mol. Biochem. Parasitol 14, 127-139. doi: 10.1016/0166-6851(85) 90032-5

Lasonder, E., Rijpma, S. R., Van Schaijk, B. C., Hoeijmakers, W. A., Kensche, P. R., Gresnigt, M. S., et al. (2016). Integrated Transcriptomic and Proteomic Analyses of P. Falciparum Gametocytes: Molecular Insight Into Sex-Specific Processes and Translational Repression. Nucleic Acids Res. 44, 6087-6101. doi: 10.1093/nar/gkw536

Liu, F., Li, L., Zheng, W., He, Y., Wang, Y., Zhu, X., et al. (2018). Characterization of Plasmodium Berghei Pbg37 as Both a Pre- and Postfertilization Antigen With Transmission-Blocking Potential. Infect. Immun. 86, e00785-17. doi: 10.1128/IAI.00785-17

Liu, Y., Tewari, R., Ning, J., Blagborough, A. M., Garbom, S., Pei, J., et al. (2008). The Conserved Plant Sterility Gene HAP2 Functions After Attachment of Fusogenic Membranes in Chlamydomonas and Plasmodium Gametes. Genes Dev. 22, 1051-1068. doi: 10.1101/gad.1656508

Liu, F., Yang, F., Wang, Y., Hong, M., Zheng, W., Min, H., et al. (2021). A Conserved Malaria Parasite Antigen Pb22 Plays a Critical Role in Male Gametogenesis in Plasmodium Berghei. Cell Microbiol. 23, e13294. doi: $10.1111 / \mathrm{cmi} .13294$

Longley, R. J., White, M. T., Takashima, E., Brewster, J., Morita, M., Harbers, M., et al. (2020). Development and Validation of Serological Markers for Detecting Recent Plasmodium Vivax Infection. Nat. Med. 26, 741-749. doi: 10.1038/ s41591-020-0841-4

Macdonald, N. J., Nguyen, V., Shimp, R., Reiter, K., Herrera, R., Burkhardt, M., et al. (2016). Structural and Immunological Characterization of Recombinant 6-Cysteine Domains of the Plasmodium Falciparum Sexual Stage Protein Pfs230. J. Biol. Chem. 291, 19913-19922. doi: 10.1074/jbc.M116.732305

Meerstein-Kessel, L., van der Lee, R., Stone, W., Lanke, K., Baker, D. A., Alano, P., et al. (2018). Probabilistic Data Integration Identifies Reliable GametocyteSpecific Proteins and Transcripts in Malaria Parasites. Sci. Rep. 8, 410. doi: 10.1038/s41598-017-18840-7

Miura, K., Deng, B., Tullo, G., Diouf, A., Moretz, S. E., Locke, E., et al. (2013a). Qualification of Standard Membrane-Feeding Assay With Plasmodium Falciparum Malaria and Potential Improvements for Future Assays. PloS One 8, e57909. doi: 10.1371/journal.pone.0057909

Miura, K., Tachibana, M., Takashima, E., Morita, M., Kanoi, B. N., Nagaoka, H., et al. (2019). Malaria Transmission-Blocking Vaccines: Wheat Germ Cell-Free Technology can Accelerate Vaccine Development. Expert Rev. Vaccines 18, 1017-1027. doi: 10.1080/14760584.2019.1674145

Miura, K., Takashima, E., Deng, B., Tullo, G., Diouf, A., Moretz, S. E., et al. (2013b). Functional Comparison of Plasmodium Falciparum TransmissionBlocking Vaccine Candidates by the Standard Membrane-Feeding Assay. Infect. Immun. 81, 4377-4382. doi: 10.1128/IAI.01056-13

Moorthy, V. S., Newman, R. D., and Okwo-Bele, J. M. (2013). Malaria Vaccine Technology Roadmap. Lancet 382, 1700-1701. doi: 10.1016/S0140-6736(13) 62238-2

Morita, M., Takashima, E., Ito, D., Miura, K., Thongkukiatkul, A., Diouf, A., et al. (2017). Immunoscreening of Plasmodium Falciparum Proteins Expressed in a Wheat Germ Cell-Free System Reveals a Novel Malaria Vaccine Candidate. Sci. Rep. 7:46086. doi: 10.1038/srep46086

Muthui, M. K., Takashima, E., Omondi, B. R., Kinya, C., Muasya, W. I., et al. (2021). Characterization of Naturally Acquired Immunity to a Panel of Antigens Expressed in Mature P. Falciparum Gametocytes. Front. Cell Infect. Microbiol. 11, 774537. doi: 10.3389/fcimb.2021.774537

Nikolaeva, D., Illingworth, J. J., Miura, K., Alanine, D. G. W., Brian, I. J., Li, Y., et al. (2020). Functional Characterization and Comparison of Plasmodium Falciparum Proteins as Targets of Transmission-Blocking Antibodies. Mol. Cell Proteomics 19, 155-166. doi: 10.1074/mcp.RA117.000036

Ntege, E. H., Takashima, E., Morita, M., Nagaoka, H., Ishino, T., and Tsuboi, T. (2017). Blood-Stage Malaria Vaccines: Post-Genome Strategies for the Identification of Novel Vaccine Candidates. Expert Rev. Vaccines 16, 769779. doi: 10.1080/14760584.2017.1341317

Outchkourov, N. S., Roeffen, W., Kaan, A., Jansen, J., Luty, A., Schuiffel, D., et al. (2008). Correctly Folded Pfs48/45 Protein of Plasmodium Falciparum Elicits Malaria Transmission-Blocking Immunity in Mice. Proc. Natl. Acad. Sci. U.S.A. 105, 4301-4305. doi: 10.1073/pnas.0800459105 
Patel, P. N., and Tolia, N. (2021). Structural Vaccinology of Malaria TransmissionBlocking Vaccines. Expert Rev. Vaccines 20, 199-214. doi: 10.1080/ 14760584.2021.1873135

Qiu, Y., Zhao, Y., Liu, F., Ye, B., Zhao, Z., Thongpoon, S., et al. (2020). Evaluation of Plasmodium Vivax HAP2 as a Transmission-Blocking Vaccine Candidate. Vaccine 38, 2841-2848. doi: 10.1016/j.vaccine.2020.02.011

Rts, S.C.T.P. (2015). Efficacy and Safety of RTS,S/AS01 Malaria Vaccine With or Without a Booster Dose in Infants and Children in Africa: Final Results of a Phase 3, Individually Randomised, Controlled Trial. Lancet 386, 31-45. doi: 10.1016/S0140-6736(15)60721-8

Sagara, I., Healy, S. A., Assadou, M. H., Gabriel, E. E., Kone, M., Sissoko, K., et al. (2018). Safety and Immunogenicity of Pfs25H-EPA/Alhydrogel, a Transmission-Blocking Vaccine Against Plasmodium Falciparum: A Randomised, Double-Blind, Comparator-Controlled, Dose-Escalation Study in Healthy Malian Adults. Lancet Infect. Dis. 18, 969-982. doi: 10.1016/S14733099(18)30344-X

Sala, K. A., Nishiura, H., Upton, L. M., Zakutansky, S. E., Delves, M. J., Iyori, M., et al. (2015). The Plasmodium Berghei Sexual Stage Antigen PSOP12 Induces Anti-Malarial Transmission Blocking Immunity Both In Vivo and In Vitro. Vaccine 33, 437-445. doi: 10.1016/j.vaccine.2014.11.038

Stone, W. J. R., Campo, J. J., Ouedraogo, A. L., Meerstein-Kessel, L., Morlais, I., Da, D., et al. (2018). Unravelling the Immune Signature of Plasmodium Falciparum Transmission-Reducing Immunity. Nat. Commun. 9, 558. doi: 10.1038/ s41467-017-02646-2

Tachibana, M., Baba, M., Takashima, E., Tsuboi, T., Torii, M., and Ishino, T. (2020). The C-Terminal Region of the Plasmodium Yoelii Microgamete Surface Antigen PyMigS Induces Potent Anti-Malarial TransmissionBlocking Immunity in Mice. Vaccine 38, 3129-3136. doi: 10.1016/ j.vaccine.2020.02.058

Tachibana, M., Ishino, T., Takashima, E., Tsuboi, T., and Torii, M. (2018a). A Male Gametocyte Osmiophilic Body and Microgamete Surface Protein of the Rodent Malaria Parasite Plasmodium Yoelii (PyMiGS) Plays a Critical Role in Male Osmiophilic Body Formation and Exflagellation. Cell Microbiol. 20, e12821. doi: $10.1111 / \mathrm{cmi} .12821$

Tachibana, M., Ishino, T., Tsuboi, T., and Torii, M. (2018b). The Plasmodium Yoelii Microgamete Surface Antigen (PyMiGS) Induces Anti-Malarial Transmission Blocking Immunity That Reduces Microgamete Motility/ Release From Activated Male Gametocytes. Vaccine 36, 7463-7471. doi: 10.1016/j.vaccine.2018.10.067

Tachibana, M., Sato, C., Otsuki, H., Sattabongkot, J., Kaneko, O., Torii, M., et al. (2012). Plasmodium Vivax Gametocyte Protein Pvs230 Is a TransmissionBlocking Vaccine Candidate. Vaccine 30, 1807-1812. doi: 10.1016/ j.vaccine.2012.01.003

Tachibana, M., Suwanabun, N., Kaneko, O., Iriko, H., Otsuki, H., Sattabongkot, J., et al. (2015). Plasmodium Vivax Gametocyte Proteins, Pvs48/45 and Pvs47, Induce Transmission-Reducing Antibodies by DNA Immunization. Vaccine 33, 1901-1908. doi: 10.1016/j.vaccine.2015.03.008

Talaat, K. R., Ellis, R. D., Hurd, J., Hentrich, A., Gabriel, E., Hynes, N. A., et al. (2016). Safety and Immunogenicity of Pfs25-EPA/Alhydrogel(R), a Transmission Blocking Vaccine Against Plasmodium Falciparum: An Open Label Study in Malaria Naive Adults. PloS One 11, e0163144. doi: 10.1371/ journal.pone.0163144

Tsuboi, T., Cao, Y. M., Hitsumoto, Y., Yanagi, T., Kanbara, H., and Torii, M. (1997a). Two Antigens on Zygotes and Ookinetes of Plasmodium Yoelii and Plasmodium Berghei That are Distinct Targets of Transmission-Blocking Immunity. Infect. Immun. 65, 2260-2264. doi: 10.1128/iai.65.6.2260-2264.1997

Tsuboi, T., Cao, Y. M., Kaslow, D. C., Shiwaku, K., and Torii, M. (1997b). Primary Structure of a Novel Ookinete Surface Protein From Plasmodium Berghei. Mol. Biochem. Parasitol 85, 131-134. doi: 10.1016/S0166-6851(96)02821-6

Tsuboi, T., Kaslow, D. C., Cao, Y. M., Shiwaku, K., and Torii, M. (1997c). Comparison of Plasmodium Yoelii Ookinete Surface Antigens With Human and Avian Malaria Parasite Homologues Reveals Two Highly Conserved Regions. Mol. Biochem. Parasitol 87, 107-111. doi: 10.1016/S0166-6851(97) 00049-2

Tsuboi, T., Kaslow, D. C., Gozar, M. M., Tachibana, M., Cao, Y. M., and Torii, M. (1998). Sequence Polymorphism in Two Novel Plasmodium Vivax Ookinete Surface Proteins, Pvs25 and Pvs28, That Are Malaria Transmission-Blocking Vaccine Candidates. Mol. Med. 4, 772-782. doi: 10.1007/BF03401770
Tsuboi, T., Tachibana, M., Kaneko, O., and Torii, M. (2003). TransmissionBlocking Vaccine of Vivax Malaria. Parasitol Int. 52, 1-11. doi: 10.1016/s13835769(02)00037-5

Van Dijk, M. R., Van Schaijk, B. C., Khan, S. M., Van Dooren, M. W., Ramesar, J., Kaczanowski, S., et al. (2010). Three Members of the 6-Cys Protein Family of Plasmodium Play a Role in Gamete Fertility. PloS Pathog. 6, e1000853. doi: 10.1371/journal.ppat.1000853

Van Schaijk, B. C., Van Dijk, M. R., Van De Vegte-Bolmer, M., Van Gemert, G. J., Van Dooren, M. W., Eksi, S., et al. (2006). Pfs47, Paralog of the Male Fertility Factor Pfs48/45, Is a Female Specific Surface Protein in Plasmodium Falciparum. Mol. Biochem. Parasitol 149, 216-222. doi: 10.1016/ j.molbiopara.2006.05.015

Vermeulen, A. N., Ponnudurai, T., Beckers, P. J., Verhave, J. P., Smits, M. A., and Meuwissen, J. H. (1985). Sequential Expression of Antigens on Sexual Stages of Plasmodium Falciparum Accessible to Transmission-Blocking Antibodies in the Mosquito. J. Exp. Med. 162, 1460-1476. doi: 10.1084/jem.162.5.1460

Vogel, G. (2021). WHO Gives First Malaria Vaccine the Green Light. Science 374, 245-246. doi: 10.1126/science.acx9344

Wang, J., Xu, C., Wong, Y. K., He, Y., Adegnika, A. A., Kremsner, P. G., et al. (2020). Preparedness Is Essential for Malaria-Endemic Regions During the COVID-19 Pandemic. Lancet 395, 1094-1096. doi: 10.1016/S0140-6736(20) 30561-4

Wang, J., Zheng, W., Liu, F., Wang, Y., He, Y., Zheng, L., et al. (2017). Characterization of Pb51 in Plasmodium Berghei as a Malaria Vaccine Candidate Targeting Both Asexual Erythrocytic Proliferation and Transmission. Malar J. 16, 458. doi: 10.1186/s12936-017-2107-2

White, M. T., Verity, R., Griffin, J. T., Asante, K. P., Owusu-Agyei, S., Greenwood, B., et al. (2015). Immunogenicity of the RTS,S/AS01 Malaria Vaccine and Implications for Duration of Vaccine Efficacy: Secondary Analysis of Data From a Phase 3 Randomised Controlled Trial. Lancet Infect. Dis. 15, 14501458. doi: 10.1016/S1473-3099(15)00239-X

Who (2020). World Malaria Report 2020 (Geneva, Switzerland: WHO Press).

Williamson, K. C., Criscio, M. D., and Kaslow, D. C. (1993). Cloning and Expression of the Gene for Plasmodium Falciparum Transmission-Blocking Target Antigen, Pfs230. Mol. Biochem. Parasitol 58, 355-358. doi: 10.1016/ 0166-6851(93)90058-6

Williamson, K. C., Keister, D. B., Muratova, O., and Kaslow, D. C. (1995). Recombinant Pfs230, a Plasmodium Falciparum Gametocyte Protein, Induces Antisera That Reduce the Infectivity of Plasmodium Falciparum to Mosquitoes. Mol. Biochem. Parasitol 75, 33-42. doi: 10.1016/0166-6851(95) 02507-3

Zheng, W., Kou, X., Du, Y., Liu, F., Yu, C., Tsuboi, T., et al. (2016). Identification of Three Ookinete-Specific Genes and Evaluation of Their TransmissionBlocking Potentials in Plasmodium Berghei. Vaccine 34, 2570-2578. doi: 10.1016/j.vaccine.2016.04.011

Zheng, W., Liu, F., He, Y., Liu, Q., Humphreys, G. B., Tsuboi, T., et al. (2017). Functional Characterization of Plasmodium Berghei PSOP25 During Ookinete Development and as a Malaria Transmission-Blocking Vaccine Candidate. Parasit Vectors 10:8. doi: 10.1186/s13071-016-1932-4

Conflict of Interest: The authors declare that the research was conducted in the absence of any commercial or financial relationships that could be construed as a potential conflict of interest.

Publisher's Note: All claims expressed in this article are solely those of the authors and do not necessarily represent those of their affiliated organizations, or those of the publisher, the editors and the reviewers. Any product that may be evaluated in this article, or claim that may be made by its manufacturer, is not guaranteed or endorsed by the publisher.

Copyright (C) 2021 Takashima, Tachibana, Morita, Nagaoka, Kanoi and Tsuboi. This is an open-access article distributed under the terms of the Creative Commons Attribution License (CC BY). The use, distribution or reproduction in other forums is permitted, provided the original author(s) and the copyright owner(s) are credited and that the original publication in this journal is cited, in accordance with accepted academic practice. No use, distribution or reproduction is permitted which does not comply with these terms. 